\title{
Urological issues arising after treatment of pediatric malignancies
}

\author{
Rodrigo L.P. Romao, MD ${ }^{1,2}$; Ashley Cox, $M D^{2}$ \\ 'Division of Pediatric Urology, Division of Pediatric Surgery, IWK Health Centre; '2Department of Urology, Dalhousie University, Halifax, NS; Canada
}

Cite as: Can Urol Assoc J 2018;12(4Suppl1):S37-41. http://dx.doi.org/10.5489/cuai.5279

\section{Introduction}

Pediatric oncology survivors are not traditionally considered when reviewing the patient groups in need of transitional care in urology. ${ }^{1,2}$ When assessing urological issues that arise related to childhood malignancies, there are two separate scenarios. First, there are children treated for genitourinary malignancies that may have ongoing or de novo urological issues to be followed by an adult urologist. Second, there are children treated for non-genitourinary malignancies that may acquire secondary urological complications, of which the adult urologist must be aware. This article will outline examples of each situation with the hopes of providing both the pediatric and adult urologist information that will ease the transition and treatment of these complex patients.

Children are now living longer following treatment of pediatric malignancies, with the overall survival rate approaching $75 \%{ }^{3}$ Delayed side effects of treatment happen to a significant proportion of patients and up to $25 \%$ may be severe or life-threatening. Many clinical sequelae of pediatric cancer treatment may not become apparent until the adult years. ${ }^{3}$

\section{Long-term complications of urological malignancies in children}

\section{Case 1}

A 33-year-old male presents with an incidentally discovered renal mass affecting his right solitary kidney. He has a past medical history of receiving treatment for Wilms tumour (WT) at the age of three. His treatment was comprised of an upfront left radical nephrectomy and lymph node sampling. Pathology confirmed favourable histology WT with positive nodes. He was treated for stage 3 disease with three-drug chemotherapy (vincristine, dactynomicyn, and doxorubicin) plus abdominal radiation with excellent response and no evidence of recurrence on followup.
WT is the most common renal tumour in childhood; the evolution of multimodal treatment has led to an overall fiveyear survival rate over $90 \%$ in recent decades. Nonetheless, treatment-related long-term morbidity is significant and relevant to adult urologists.

The burden of chronic health conditions and death was significantly higher in a 25-year followup of 1256 WT survivors enrolled in the Childhood Cancer Survivor Study, compared to a sibling control population. ${ }^{4}$ The most common causes of death were secondary malignancies, recurrent disease, and cardiac events. Specific urological morbidity data were not provided on this study, however, a case of death related to urinary tract disease was mentioned. Death was also more common in patients exposed to radiation and doxorubicin.

The most common secondary malignancies in WT survivors are soft tissue sarcomas and breast cancer; ${ }^{4}$ nevertheless, case reports of renal cell carcinoma (RCC) and other renal tumours arising in young adult patients previously treated for WT have been published..$^{5,6}$ This is exceptionally pertinent to the case presented, as the treating urologist will be faced with a tumour affecting a previously irradiated, solitary kidney that was exposed to chemotherapy.

As with any case of renal masses and related surgery in adulthood, careful consideration about preservation of renal function is also imperative in this case. Although the cumulative incidence of end-stage renal disease in survivors of unilateral WT after 20 years is low $(<1 \%),{ }^{7}$ reduced glomerular filtration rates (GFR) compatible with lower categories of chronic kidney disease (CKD) have been reported in WT survivors, with a higher risk in those exposed to radiation. ${ }^{8,9}$

The discussion about renal function and its preservation becomes even more applicable to survivors of bilateral WT. The recommended treatment for such patients is preoperative chemotherapy followed by bilateral nephron-sparing surgery (NSS) whenever possible. A recent Children's Oncology Group (COG) publication focused on almost 200 patients with bilateral tumours showed that $39 \%$ retained parts of both kidneys, although most (48\%) were still treated with unilateral total and contralateral partial nephrectomy, with excellent survival rates (overall survival [OS] 94\%). ${ }^{10}$ Patients with bilateral disease are at much higher risk for renal dysfunction, with 
rates of $4 \%$ and $19 \%$ in long-term followup for synchronous and metachronous tumours, respectively. ${ }^{7}$ Pediatric oncology survivorship programs now measure GFR routinely as part of the long-term followup, therefore, such information should be obtainable by adult practitioners involved in the care of WT survivors. Hence, urologists planning renal surgery in WT survivors should expect a higher susceptibility to renal dysfunction and plan accordingly.

Additionally, from a preoperative planning standpoint, urologists should be aware that doxorubicin is cardiotoxic; therefore, patients exposed to it in infancy should have their cardiac function assessed before undergoing anesthesia and surgery.

In summary, nephrotoxicity from surgery, chemotherapy, and radiation exposes WT survivors to a multitude of risks in adulthood that carry significance to the practicing adult urologist. Whether presenting with a specific problem, such as the example above, or more generic issues, such as urinary tract infections (UTI) or stones, it is important to bear in mind the intrinsic nuances of surviving pediatric urological cancer.

\section{Case 2}

A 20-year-old male is transitioned for care from a pediatric urologist. He has ongoing bladder function issues associated with a past medical history of prostate rhabdomyosarcoma (RMS) treated with chemotherapy and radiation at age four.

RMS is the third most common solid, extracranial tumour of infancy and the most common type of soft tissue sarcoma affecting children. Location of the primary tumour carries prognostic significance. Common locations include head and neck, extremities, trunk and genitourinary. Within the genitourinary system, which harbours $15-20 \%$ of all RMS, bladder and prostate lesions are considered unfavourable locations, while paratesticular and vaginal primaries are considered favourable sites and, as such, are associated with a better prognosis.

Historically, the cure rates of RMS were approximately $20 \%$ with surgery alone. ${ }^{11}$ The much-improved survival with multimodal therapy means that more patients will be living into adulthood following treatment, including pelvic surgery, chemotherapy, and radiation therapy, all of which may result in urological complications.

The most relevant aspect of bladder/prostate RMS treatment in childhood is the long-term impact of local therapy on the genitourinary tract. Although the OS for localized disease exceeds $70 \%$, there is clear evidence that failure to administer therapy aimed at achieving local control to the primary site (surgery or radiation) is associated with decreased event-free survival. ${ }^{12}$ Bladder-preserving strategies are always encouraged in the treatment of these patients, but radiation to the young pelvis also carries significant long-term morbidity to the organs in the radiation field, including the bladder.

Current COG protocols advocate for treatment with chemotherapy and radiotherapy, unless surgical resection is feasible without complete organ loss (i.e., partial cystectomy for tumour at the dome). Very few studies have reported formal functional assessment of the genitourinary system in RMS survivors. Arndt et al reported that $40 \%$ of survivors from the Intergroup RMS Study IV reported normal bladder function based on history alone, at a median followup of six years. ${ }^{13}$ Martelli et al evaluated quality of life at a median followup of 10 years after treatment in 27 patients with bladder/prostate RMS treated with a combination of non-radical surgery and brachytherapy; they reported that $76 \%$ considered themselves as having a normal QOL. Nonetheless, 11 patients underwent urodynamic studies with abnormal findings, most notably detrusor-sphincter dyssynergia. ${ }^{14}$ Another recent study documents a high incidence of erectile dysfunction (ED) in bladder/prostate RMS survivors, which responded to intra-cavernous injection. ${ }^{15}$

\section{Non-genitourinary malignancy with urological complications}

\section{Case 3}

A 29-year-old female was referred with an episode of pyelonephritis with associated hydronephrosis. Past medical history was significant for metastatic pelvic RMS treated at age 12. The RMS was located in the ischiorectal fossa posterior to the bladder, lateral to the rectal wall, extending into the right upper thigh. Biopsy revealed embryonal RMS. She was treated with chemoradiation, including cyclophosphamide.

This patient was found to have severe lower urinary tract symptoms (LUTS) due to radiation cystitis and recurrent episodes of pyelonephritis, often requiring visits to the emergency department. Ultrasonography revealed mild right-sided hydronephrosis. Creatinine was normal. Computed tomography $(\mathrm{CT})$ scan showed a $5 \mathrm{~mm}$ distal right ureterovesical junction stone. Renal scan revealed no evidence of obstruction, with differential function of $37 \%$ on the right and $63 \%$ on the left side. Cystoscopy showed evidence of radiation cystitis. Retrograde pyelogram on the right showed quite marked stenosis consistent with a stricture from previous radiation. The stone was proximal to this area. Unable to perform ureteroscopy due to the stricture, a stent was placed to help dilate the stenosed segment of ureter. The stent was extremely bothersome to patient due to pain and hematuria. After two weeks, ureteroscopy was performed and the stone retrieved, however, the patient was very distraught at the need for any additional diagnostic imaging or procedure involving radiation due to her medical history. 


\section{Complications of treatment}

There are several challenges to transitioning patients following treatment of pediatric malignancies. First, we know several urological complications may occur following treatment of pediatric malignancies due to the effects of surgery, chemotherapy, and radiation (Table 1). These complications include, but are not limited to: bladder dysfunction, radiation cystitis, urinary incontinence, urethral stenosis, ureteral stricture, hydronephrosis, renal failure, fertility issues, and secondary bladder cancers. Bladder dysfunction is common following multimodal treatment for pelvic RMS. ${ }^{16}$ Raney et al assessed complications of treatment for RMS after a median followup of eight years (range 3-24) and found that 31\% had urinary incontinence, 55\% had recurrent UTIs, 40\% had decreased renal function, and $25 \%$ had chronic hematuria. ${ }^{17}$

Radiation induces an oxidative stress injury to cells resulting in cell death. The addition of chemotherapy interferes with the cells' ability to regenerate. Over time, this leads to vascular damage, ischemia, and chronic tissue fibrosis. ${ }^{18}$ As mentioned above, radiation therapy can lead to several urological complications, including infertility, radiation cystitis, ureteric strictures, and secondary malignancies. After radiation therapy, tissue is more prone to infection, poor healing, and fistula development. This presents another challenge in the management of childhood cancer survivors. In the case presented here, treatment of the patient's stone was complicated by a radiation-induced ureteric stricture.

Male childhood cancer survivors are also at increased risk for ED. In a study focused on the Childhood Cancer Survivor Cohort, survivors had less sexual activity and a 2.6-fold increase in ED based on validated questionnaires compared to their healthy siblings. Exposure to testicular radiation, pelvic, prostate, or spinal surgery had a higher risk of ED. ${ }^{19}$

Fertility issues arise may arise in both male and female patients following chemotherapy or radiation therapy. ${ }^{20,21}$ RMS survivors are particularly at risk due to exposure to high doses of alkylating agents (cyclophosphamide) and pelvic radiation. Several chemotherapeutic agents have been linked to infertility, including: busulfan, carboplatin, cisplatin, cyclophosphamide, etoposide, ifosfamide, melphalan, and procarbazine. ${ }^{20}$ As adults, male patients may be referred to urology for further investigations and management of infertility. Some recommend that all post-pubertal males diagnosed with a malignancy be offered sperm cryopreservation prior to undergoing chemotherapy, regardless of the gonadotoxicity of the agents. ${ }^{21}$ This is, however, controversial, as some patients may incur significant costs for a very low risk. For male patients who have successfully cryopreserved sperm, assisted reproductive techniques, such as intracytoplasmic sperm injection, increases the likelihood of successful pregnancy. Feldschuh et al report two live births following intrauterine insemination with sperm that was cryopreserved for 28 years. ${ }^{22}$

\begin{tabular}{|c|c|}
\hline Urological issues & Risk factors \\
\hline Infertility & $\begin{array}{l}\text { - Exposure to alkylating } \\
\text { chemotherapeutic agents } \\
\text { (e.g., cyclophosphamide) } \\
\text { - } \text { Testicular surgery } \\
\text { - Radiation exposure }\end{array}$ \\
\hline Erectile dysfunction & $\begin{array}{l}\text { - Testicular radiation } \\
\text { - Surgery on bladder, prostate, or spinal } \\
\text { cord in childhood }\end{array}$ \\
\hline Renal dysfunction & - Renal surgery and radiation \\
\hline $\begin{array}{l}\text { Radiation cystitis, } \\
\text { bladder dysfunction }\end{array}$ & - Pelvic radiation, surgery \\
\hline $\begin{array}{l}\text { Secondary } \\
\text { malignancies (renal } \\
\text { cell carcinoma) }\end{array}$ & $\begin{array}{l}\text { - Exposure to platinum-based } \\
\text { chemotherapy } \\
\text { - Diagnosis of neuroblastoma, Wilms } \\
\text { tumour? } \\
\text { - Radiation exposure }\end{array}$ \\
\hline
\end{tabular}

Pediatric cancer survivors are at increased risk of secondary malignancies for several reasons. Pelvic and abdominal radiation therapy leads to an increased risk of developing a secondary malignancy. Patients undergoing pelvic radiation for gynecological malignancies are at increased risk for secondary cancers of bowel, bladder, and genital sites. Survivors of testicular cancer are at an increased risk of digestive and genitourinary tract malignancies. ${ }^{23}$ Furthermore, cyclophosphamide and related chemotherapeutic agents are thought to be risk factors for the development of urothelial cell carcinoma. Pediatric patients diagnosed and treated for malignancy are frequently exposed to repeat diagnostic imaging, such as positron emission(PET)/ CT scans, nuclear medicine scans, and fluoroscopy. The cumulative radiation dosage received varies depending on the type of underlying malignancy, ${ }^{24}$ but seems to be significant. ${ }^{25}$ As children are more sensitive to radiation-induced carcinogenesis and have many remaining years of life to develop a secondary malignancy, repeat CT scans in childhood are thought to increase the risk of secondary malignancy. ${ }^{26}$ In addition, as these patients enter adulthood, other risk factors for malignancy must also be considered, such as smoking, human papillomavirus status, and genetic predisposition.

With the increasing number of pediatric cancer survivors, it is likely that urologists will witness a rise in the number of urological complications related to the treatment of pediatric malignancies. ${ }^{3}$ Furthermore, adult urologists should know that a possible increased risk of RCC in childhood cancer survivors has been described. Wilson et al reported a higher standardized incidence ratio (SIR 8; confidence interval [CI] 5.2-11.7) of RCC in a cohort of over 14000 childhood cancer survivors compared to the general population, at a median followup of 22 years; a diagnosis of neuroblastoma, radiation to the kidney $\geq 5 \mathrm{~Gy}$, and exposure to platinumbased chemotherapy were identified as risk factors. ${ }^{27}$ 


\section{The transition process}

Although these patients don't fall within the usual group of urology transition patients, several unique challenges exist when managing adult patients with complicated pediatric histories. Patients themselves may not be aware of the details of their medical history and their parents may not be available to provide collateral history. The underlying diagnosis, risk of recurrence (if any), and details of treatment may be difficult to ascertain. Often, current treatment modalities for pediatric cancers will differ than the treatment given many years ago, complicating the matter further. In a recent nationwide Korean survey study, Ahn et al found that more than half of parents of childhood cancer survivors were not aware of the exposure to cardiotoxic agents $(72.9 \%)$ or radiation therapy $(56.3 \%)$. This study also found that providing information regarding the long-term side effects and prevention of secondary cancers was correlated with more concern and more followup visits, but didn't necessarily correlate with an increase in awareness of side effects. ${ }^{28}$

Similar to patients being transitioned with congenital anomalies, we are seeing this group of patients living longer into adulthood. It will take time to fully understand the risks associated with these pediatric treatments. Meanwhile, it remains difficult to provide patients and caregivers with information on what to expect in the future with regards to complications related to cancer treatment.

In medicine, the definition of transition is the process of moving a patient from pediatric to adult care. Ideally, there should be a specific period of "transition;" however, this may not be feasible in patients surviving pediatric malignancies, as complications may not arise at all and the risk is difficult to predict.

\section{Recommendations}

In order to manage the challenges of transition of patients who have survived pediatric malignancies, it is important to understand the underlying condition for which they were treated and the treatment modalities used. There is a paucity of literature to guide the adult urologist on how to manage patients who have survived pediatric malignancies. The rate that these complications occur beyond childhood is unknown; therefore, it is not possible to determine if routine assessment is necessary.

When a patient is referred with a urological issue related to a previous childhood malignancy, several recommendations can be made. First, it is recommended to contact the pediatric cancer centre and/or hospital where the patient was treated, obtain old operative notes and diagnostic imaging reports, contact family physicians, and obtain collateral history from family if necessary. Each patient requires an individualized approach. The COG long-term followup guide- lines for survivors of childhood, adolescent, and young adult cancers may help provide some guidance on how to survey this patient group for ongoing late effects. These guidelines are easy to read, risk-based, exposure-related clinical practice guidelines. ${ }^{3}$ (http://www.survivorshipguidelines.org/)

In general, a detailed history is necessary to assess symptoms such as bothersome LUTS, incontinence, UTIs, hematuria, stones, fertility issues, and sexual dysfunction (male and female). A thorough physical exam is recommended to assess for pain, surgical scars, radiation markings, radiation damage, vaginal atrophy, testicular atrophy, incontinence, and signs of secondary malignancy. Urine studies and bloodwork, including urinalysis, urine culture, renal function assessment, testosterone, prostate-specific antigen, semen analysis, etc., may be required. Investigations, such as imaging studies, cystoscopy, and urodynamic studies, may be required. Care should be taken to minimize additional radiation through imaging and procedures.

\section{What to tell the patient?}

As the adult urologist, it is important to inform the patient of the potential risk of urological complications from previous treatment. The COG has published "Health Links" for patients. This includes patient-appropriate information on bladder health, cystectomy, kidney health, neurogenic bladder, and single kidney health (http://www.survivorshipguidelines.org/). Furthermore, there are also resources related to male and female reproductive issues. Patients should be advised on whether or not ongoing surveillance is necessary and in what form (i.e., annual urinalysis to check for microscopic hematuria or surveillance cystoscopy to assess for bladder tumours). Unfortunately, at this point in time, an evidence-based approach to this clinical decision-making is not feasible.

In addition to communication with the patient, the urologist should also focus on communication with other treating physicians, such as family physicians and other specialists who may be managing related complications. In turn, this will promote a shared-care approach due to the complexity of some of these patients.

Competing interests: Dr. Cox has attended advisory boards, is a speaker for and has received payment/honoraria from Astellas and Pfizer; and has participated in a clinical trial supported by Aquinox. Dr. Romao reports no competing personal or financial conflicts.

This paper has been peer reviewed. 


\section{References}

1. Lambert SM. Transitional care in pediatric urology. Semin Pediatr Surg 2015;24:73-8. https://doi.org/10.1053/i.sempedsurg.2015.01.004

2. Timberlake MD, Corbett ST, Costabile RA, et al. Identification of adolescent and adult patients receiving pediatric urologic care and establishment of a dedicated transition clinic. J Pediatr Urol 2015;11:62el-6.

3. Landier W, Bhatia S, Eshelman DA, et al. Development of risk-based guidelines for pediatric cancer survivors: The Children's Oncology Group long-term followup guidelines from the Children's Oncology Group Late Effects Committee and Nursing Discipline. J Clin Oncol 2004;22:4979-90. https://doi.org/10.1200/JC0.2004.11.032

4. Termuhlen AM, Tersak JM, Liv Q, et al. Twenty-five-year followup of childhood Wilms tumour: A report from the Childhood Cancer Survivor Study. Ped Blood Cancer 2011;57:1210-6. https://doi.org/10.1002/ pbc. 23090

5. Rich BS, McEvoy MP, La Quaglia MP. A case of renal cell carcinoma after successful treatment of Wilms tumour. J Pediatr Surg 2010;45:1883-6. https://doi.org/10.1016/i.jpedsurg.2010.06.032

6. Cherullo EE, Ross JH, Kay R, et al. Renal neoplasms in adult survivors of childhood Wilms tumour. J Urol 2001;165:2013-6. https://doi.org/10.1016/S0022-5347(05)66283-0

7. Lange J, Peterson SM, Takashima JR, et al. Risk factors for end-stage renal disease in non-WTi-syndromic Wilms tumour. J Urol 2011;186:378-86. https://doi.org/10.1016/i.juro.2011.03.110

8. Green DM. Evaluation of renal function after successful treatment for unilateral, non-syndromic Wilms tumour. Ped Blood Cancer 2013;60:1929-35. https://doi.org/10.1002/pbc.24738

9. Romao RLP, Lorenzo AJ. Renal function in patients with Wilms tumour. Urol Oncol 2016;34:33-41. https://doi.org/10.1016/i.urolonc.2015.07.002

10. Ehrlich P, Chi YY, Chintagumpala MM, et al. Results of the first prospective, multi-institutional treatment study in children with bilateral Wilms tumour (AREN0534): A report from the Children's Oncology Group. Ann Surg 2017;266:470-8. https://doi.org/10.1097/SLA.0000000000002356

11. Sutow WW, Sullivan MP, Ried HL, et al. Prognosis in childhood rhabdomyosarcoma. Cancer 1970;25:138490. https://doi.org/10.1002/1097-0142(197006)25:6<1384::AlD-CNCR2820250617>3.0.C0;2-T

12. Rodeberg DA, Anderson JR, Arndt CA, et al. Comparison of outcomes based on treatment algorithms for rhabdomyosarcoma of the bladder/prostate: Combined results from the Children's Oncology Group, German Cooperative Soft Tissue Sarcoma Study, Italian Cooperative Group, and International Society of Pediatric Oncology Malignant Mesenchymal Tumours Committee. Int I Cancer 2011;128:1232-9. https://doi.org/10.1002/iic.25444

13. Arndt C, Rodeberg D, Breiffeld PP, et al. Does bladder preservation (as a surgical principle) lead to retaining bladder function in bladder/prostate rhabdomyosarcoma? Results from intergroup rhabdomyosarcoma study iv. J Urol 2004;171:2396-403. https://doi.org/10.1097/01.ju.0000127752.41749.a4

14. Martelli H, Borrego P, Guérin F, et al. Quality of life and functional outcome of male patients with bladder-prostate rhabdomyosarcoma treated with conservative surgery and brachytherapy during childhood. Brachytherapy 2016;15:306-11. https://doi.org/10.1016/i.brachy.2016.01.001
15. Frees $S$, Rubenwolf $P$, Ziesel $C$, et al. Erectile function after treatment for rhabdomyosarcoma of prostate and bladder. J Pediatr Urol 2016;12:404.el-6. https://doi.org/10.1016/i.jpurol.2016.07.002

16. Atra A, Ward HC, Aitken K, et al. Conservative surgery in multimodal therapy for pelvic rhabdomyosarcoma in children. Br J Cancer 1994;70:1004-8. https://doi.org/10.1038/bic.1994.438

17. Raney $B$, Anderson J, Jenney $M$, et al. Late effects in 164 patients with rhabdomyosarcoma of the bladder/prostate region: A report from the international workshop. J Urol 2006;176:2190-4. https://doi.org/10.1016/i.juro.2006.07.064

18. Morris KA, Haboubi NY. Pelvic radiation therapy: Between delight and disaster. World J Gastrointest Surg 2015;7:279-88. htrps://doi.org/10.4240/wigs.v7.i11.279

19. Ritenour C, Seidel K, Leisenring W, et al. Erectile dysfunction in male survivors of childhood cancer — a report from the Childhood Cancer Survivor Study. J Sex Med 2016;13:945-54. https://doi.org/10.1016/i.jsxm.2016.03.367

20. Reinmuth $S$, Hohmann $C$, Rendtorff $R$, et al. Impact of chemotherapy and radiotherapy in childhood on fertility in adulthood: The FeCt-survey of childhood cancer survivors in Germany. J Cancer Res Clin Oncol 2013;139:2071-8. https://doi.org/10.1007/s00432-013-1527-9

21. Ginsberg JP. Educational paper: The effect of cancer therapy on fertility, the assessment of fertility and fertility preservation options for pediatric patients. Eur I Pediatr 2011;170:703-8. hitps://doi.org/10.1007/s00431-010-1359-4

22. Feldschuh J, Brassel J, Durso N, et al. Successful sperm storage for 28 years. Fertil Steril 2005;84:1017. https://doi.org/10.1016/i.fertnstert.2005.05.015

23. Braunstein S, Nakamura JL. Radiotherapy-induced malignancies: Review of clinical features, pathobioogy, and evolving approaches for mitigating risk. Front Oncol 2013;3:73. https://doi.org/10.3389/ fonc.2013.00073

24. Ahmed BA, Connolly BL, Shroff P, et al. Cumulative effective doses from radiologic procedures for pediatric oncology patients. Pediatrics 2010;126:e851-8. https://doi.org/10.1542/peds.2009-2675

25. Chawla SC, Federman N, Zhang D, et al. Estimated cumulative radiation dose from PET/CT in children with malignancies: A 5-year retrospective review. Pediatr Radiol 2010;40:681-6. https://doi.org/10.1007/ s00247-009-1434-z

26. Miglioretti DL, Johnson E, Williams A, et al. The use of computed tomography in pediatrics and the associated radiation exposure and estimated cancer risk. JAMA Pediatr 2013;167:700-7. https://doi.org/10.1001/jamapediatrics.2013.311

27. Wilson C, Ness K, Neglia J, et al. Renal carcinoma after childhood cancer: A report from the childhood cancer survivor study. J Natl Cancer Inst 2013;105:504-8. https://doi.org/10.1093/inci/dit014

28. Ahn E, Park HJ, Baek HJ, et al. Awareness about past diagnosis and treatment history: Nationwide survey of childhood cancer survivors and their parents. Jpn J Clin Oncol 2017;47:962-8. https://doi.org/10.1093/iico/hyx102

Correspondence: Dr. Ashley Cox, Department of Urology; Dalhousie University, Halifax, NS, Canada; ashleycox@Dal.Ca 